\title{
A secondary analysis of testosterone and electrically evoked resistance training versus testosterone only (TEREX-SCI) on untrained muscles after spinal cord injury: a pilot randomized clinical trial
}

\author{
Ashraf S. Gorgey $\mathbb{1}^{1,2} \cdot$ Sally M. Abilmona ${ }^{1} \cdot$ Adam Sima $^{3} \cdot$ Refka E. Khalil ${ }^{1} \cdot{\text { Rehan } \text { Khan }^{4} \cdot \text { Robert A. Adler }}^{5,6}$
}

Received: 2 March 2019 / Revised: 10 September 2019 / Accepted: 30 September 2019 / Published online: 22 October 2019

(c) The Author(s), under exclusive licence to International Spinal Cord Society 2019

\begin{abstract}
Study design Secondary analysis of a clinical trial.

Objectives To perform a secondary analysis on the effects of neuromuscular electrical stimulation resistance training (RT) combined with testosterone replacement therapy (TRT) compared with TRT on the untrained muscles after spinal cord injury (SCI).

Setting Medical research center.

Methods Twenty-two men with chronic motor complete SCI were randomized into TRT + RT group $(n=11)$ or TRT group $(n=11)$. Both groups received 16 weeks of TRT $(2-6 \mathrm{mg} / \mathrm{day})$ via testosterone patches. The TRT + RT group received twice weekly progressive RT of the knee extensor muscles using electrical stimulation and ankle weights. Magnetic resonance images were captured to measure cross-sectional areas (CSAs) of trunk, glutei, and leg muscles.

Results Total and absolute gluteus maximus $\mathrm{m}$. $(14 \%, P=0.003$ and $16 \%, P=0.001)$, gluteus medius $\mathrm{m}$. $(10 \% ; P=0.008$ and $14 \% ; P=0.02)$, and total glutei $\mathrm{m} .(8 \%, P=0.01$ and $11 \%, P=0.005)$ CSAs increased overtime for the TRT + RT group. Mean between-group differences of 2.86 (95\% CI: 0.30, 5.4), 1.89 (95\% CI: 0.23, 3.58) and 5.27 (95\% CI: 0.90, $9.69) \mathrm{cm}^{2}$ were noted for absolute gluteus maximus, total gluteus medius and total glutei CSAs, respectively $(P<0.05)$. Trunk muscle CSAs showed a trend towards an interaction between groups.

Conclusions RT combined with low-dose TRT results in significant hypertrophy compared with TRT only on the adjacent untrained glutei muscles. Trunk muscles may require direct stimulation to evoke hypertrophy. These exploratory findings may be of clinical relevance in the reduction of incidence and severity of pelvic pressure injuries.
\end{abstract}

Ashraf S. Gorgey

ashraf.gorgey@va.gov

1 Spinal Cord Injury and Disorders, Hunter Holmes McGuire VA Medical Center, Richmond, VA, USA

2 Department of Physical Medicine and Rehabilitation; School of Medicine, Virginia Commonwealth University Richmond, Richmond, VA, USA

3 Department of Biostatistics, School of Medicine Virginia Commonwealth University, Richmond, VA, USA

4 Radiology Service, Hunter Holmes McGuire VA Medical Center, Richmond, VA, USA

5 Endocrinology Section Hunter Holmes McGuire VA Medical Center, Richmond, VA, USA

6 Endocrine Division, School of Medicine Virginia Commonwealth University, Richmond, VA, USA

\section{Introduction}

Recent guidelines have recommended that 2-3 days per week of moderate to vigorous physical activity may be necessary to attenuate secondary comorbidities after spinal cord injury (SCI) [1]. However, the guidelines did not address the extensive muscle atrophy and how this may accelerate the risks of developing secondary comorbidities [2-5]. Resistance training (RT) has been shown to be an effective rehabilitation approach in mitigating muscle atrophy, infiltration of intramuscular fat (IMF), evoking muscle hypertrophy, and improving the metabolic profile in healthy individuals, elderly populations, and in persons with SCI [6-14]. Such changes are likely to reduce the heightened risks of developing serious health consequences including cardiovascular disease and type II diabetes [4, 5]. In persons 
with motor complete (AIS A or B) SCI (i.e., unable to voluntary contract their muscles below the level of injury), electrically evoked RT using neuromuscular electrical stimulation (NMES-RT) resulted in a robust muscle hypertrophy for the trained knee extensor [7, 11, 12]. One study demonstrated $40 \%$ increase in skeletal muscle size following 12 weeks of NMES-RT in persons with SCI [8]. Another study demonstrated an increase in whole thigh, knee extensor, and flexor cross-sectional area (CSA) by $28 \%, 35 \%$, and $16 \%$, respectively, following 12 weeks of NMES-RT [7]. These studies used Dudley's protocol that relied on progressively loading the paralyzed muscle twice weekly using NMES and ankle weights [10]. The combinatory effect of 16 weeks of NMES-RT in conjunction with low-dose testosterone replacement therapy (TRT) on cardiometabolic risk factors was recently completed [11]. Lowdose TRT and electrically evoked RT resulted in $43 \%$ hypertrophy of the trained knee extensors, $14-17 \%$ increase in basal metabolic rate and decrease in visceral adiposity compared with TRT only [11].

Previous studies using electrically evoked RT have been directed toward knee extensors considering the extensive muscle atrophy that may exceed $40-50 \%$ of the original muscle size [2, 3]. Progressive RT programs have also been shown to promote hypertrophy of the surrounding untrained muscle groups [12, 13]. However, it is still unclear whether NMES-RT of the knee extensors may induce similar hypertrophy of the untrained muscles (i.e., not directly exposed to electrical stimulation) in persons with SCI. This is highly important considering the wide spectrum of complications associated with muscle atrophy in the lower extremities including blood clotting, venous pooling [15], and sacral pressure injuries following changes in the size and the quality of the glutei muscles $[16,17]$. Furthermore, Abilmona and Gorgey noted that increases in trunk muscle CSA may be associated with decreases in visceral adiposity and improvement in the metabolic profile of persons with SCI [18]. Therefore, it is possible to hypothesize that an increase in lower leg, glutei, and trunk muscle CSAs could attenuate several of the aforementioned complications.

In attempt to test this hypothesis, the effect of electrically evoked RT on the untrained trunk muscles was previously conducted [12]. The hypothesis was based on the fact that the untrained trunk muscles have close proximity to the NMES current, anatomically serving as stabilizers during NMES-induced leg extension or may be responsive to increased circulating insulin growth factors [7, 12]. The findings indicated that NMES-RT resulted in hypertrophy of knee extensors, flexors, and hip adductors but without changes in the trunk muscles following 12 weeks of training [12]. A possible explanation is the short duration of application (12 weeks) and limited endogenous growth factors that may maximize applications of NMES-RT on untrained muscles.

Persons with SCI suffer an average $43 \%$ decline in serum testosterone levels. Low serum testosterone $(<325 \mathrm{ng} / \mathrm{dL})$ has been observed in 40-60\% of men with SCI [19-21]. Recently, strong associations were noted between serum testosterone and cardiometabolic risk factors in the persons with SCI [20]. High circulating testosterone is associated with increased leg lean mass, thigh muscle CSA, and decreased visceral adiposity [20]. In fact, those with lowlevels of circulating testosterone have $72 \%$ greater visceral adiposity than those with normal testosterone [20]. TRT has been shown to be a safe and effective strategy in improving lean tissue mass and basal metabolic rate in men with SCI $[21,22]$. Therefore, the addition of low-dose TRT may maximize the effects of NMES-RT on the proximal (trunk and glutei) and distal (lower leg) untrained muscles during training of the knee extensor muscle group.

Therefore, we have expanded the analysis on recently published work to investigate the effects of low-dose TRT and NMES-RT on untrained muscles [11]. The primary objective of the current pilot study was to examine the additive effect of low-dose TRT to NMES-RT for 16 weeks on the untrained trunk, glutei, and lower leg muscles compared with TRT only in the persons with motor complete SCI. Our hypothesis was that the addition of testosterone would amplify the effects of NMES-RT on the CSAs of untrained muscles (lower leg, glutei, and trunk) when compared with TRT only in persons with chronic motor complete SCI.

\section{Methods}

\section{Participants}

Twenty-two men with chronic ( $\geq 1$ year post injury) motor complete (AIS A or B) SCI between the ages of 18 and 50 participated in the current study (NCT01652040). Recruitment started in August 2012 and ended in April 2015 after meeting the target sample size [14]. Inclusion/exclusion criteria have previously been published in detail elsewhere $[11,14]$. After providing written consent which was approved by a local ethics committee, a physical examination was conducted on each subject by a certified physician. Participants were then randomized in a blinded manner into one of the two groups either TRT and NMES-RT (TRT + RT) or TRT only for 16 weeks (Table 1).

Magnetic resonance imaging (MRI; 1.5T; General Electric) was performed at baseline (BL, week 0) and post intervention (PI, week 17) of the trunk, glutei and lower leg muscles. MRI images were captured to measure the CSA of specific muscle groups and IMF within each muscle group 
Table 1 Participants' physical and SCI characteristics for the TRT + RT and TRT groups. Values are presented as means \pm SD

\begin{tabular}{lll}
\hline & TRT + RT $(n=11)$ & TRT $(n=11)$ \\
\hline Age (years) & $37 \pm 12$ & $35 \pm 8$ \\
Height (m)_BL & $1.8 \pm 7$ & $1.8 \pm 5$ \\
Height (m)_PI & $1.8 \pm 7$ & $1.8 \pm 5$ \\
Weight $(\mathrm{kg})$-BL & $80.5 \pm 16$ & $77.6 \pm 10$ \\
Weight $(\mathrm{kg})$-PI & $83.1 \pm 16$ & $78.8 \pm 10$ \\
BMI $\left(\mathrm{kg} / \mathrm{m}^{2}\right)$-BL & $25 \pm 4.5$ & $24.0 \pm 3.4$ \\
BMI $\left(\mathrm{kg} / \mathrm{m}^{2}\right)$-PI & $25 \pm 4.7$ & $24.0 \pm 3.4$ \\
LOI & $\mathrm{C} 5-\mathrm{T} 11$ & $\mathrm{C} 6-\mathrm{T} 11$ \\
TSI (years) & $10 \pm 9$ & $7 \pm 6$ \\
ISNCSCI classification & A $(n=8) \mathrm{B}(n=3)$ & A $(n=8) \mathrm{B}(n=3)$ \\
\hline
\end{tabular}

There were no statistical differences in physical and SCI characteristics between groups at baseline

$B L$ baseline (immediately prior to 16 -week interventions), $B M I$ body mass index, ISNCSCI International Standards for Neurological Classification of Spinal Cord Injury, LOI level of injury, PI post intervention, $R T$ resistance training, TSI time since injury

(i.e., absolute muscle CSA). The recently published primary outcomes of the clinical trial were knee extensor and whole thigh muscle CSA [11]. Three untrained muscle group CSAs (trunk, glutei, and leg) were considered secondary outcome variables of the current study (see Table 2). The trunk muscle group included six individual muscles (see below), the glutei group included the total glutei, gluteus maximus and gluteus medius muscles and the lower leg included the soleus (SOL), gastrocnemius (GAS) and tibialis anterior (TA) muscles.

\section{Interventions}

\section{Testosterone replacement therapy (TRT)}

Each participant received a low dose of $2-6 \mathrm{mg} / \mathrm{day}$ of testosterone administered through transdermal testosterone patches (Androderm; Watson Pharma, Parsippany, NJ) and alternated between both shoulders [11, 14]. Participants were instructed to place the patches before bedtime on dry skin and to temporarily remove patches during bathing. BL measurements of serum testosterone were used to determine initial dosages, and subjects with $<300,300-600$, or $>600 \mathrm{ng} / \mathrm{dL}$ received 6,4 , or $2 \mathrm{mg} /$ day dosages, respectively. Participants or caregivers were instructed to count the used patches and provided a log-form to report adherence on a monthly basis to the study team. Monthly measurements of serum testosterone were monitored in a blinded fashion by an endocrinologist to adjust for the dose. If the participant's serum testosterone levels exceeded $1000 \mathrm{ng} / \mathrm{dL}$ during testing, a decreased dosage of $2 \mathrm{mg} /$ day was recommended. Participants with serum testosterone concentrations $<250 \mathrm{ng} / \mathrm{dL}$ received reeducation regarding their procedure for administering TRT and whether the patches were placed correctly or not.

\section{Resistance training (RT)}

Participants randomized in the TRT $+\mathrm{RT}$ group received progressive resistance exercise of the knee extensor muscle groups [10-14]. Twice weekly for the 16-week period, a research team member conducted exercise sessions for both lower extremities starting with the right leg and followed by the left leg. Blood pressure and heart rate were monitored through the entire session. Electrical stimulation was administered using large $(8 \times 10 \mathrm{~cm})$ electrodes that were placed laterally on the proximal thigh and medially on the distal thigh. Participants remained in their wheelchair while their knee extensors were stimulated through manually adjusted NMES current (Theratouch 4.7; Richmar, Inola, OK; Biphasic waveform, $30 \mathrm{~Hz}, 450 \mu$ s pulse width, current intensity enough to elicit full knee extension) for four sets of ten repetitions. During the first week, no ankle weights were used during training. If the participant completed all exercise sets without fatigue, weights were increased in 2pound increments. Progression in current amplitude and ankle weights were recorded and monitored throughout the 16-week intervention [11].

\section{Magnetic resonance imaging}

Participants were stationed in a supine position with their lower extremities strapped to minimize spontaneous involuntary movements due to muscle spasms. Images were captured with Echelon RAPID Torso/Body Coil (Hitachi Medical Systems America, Twingsburg, $\mathrm{OH}$ ). The technician ensured that lower portion of the coil was placed at the symphysis pubis both BL and PI for each participant $[18,20]$. A fast spin-echo sequence was used to perform T1weighted imaging (axial in-phase (IP) /out-phase (OP) with a repetition time of $140 \mathrm{~ms}$ and echo time of 4.2 and $2 \mathrm{~ms}$ for the IP and the OP, respectively; a $42-\mathrm{cm}$ field of view, matrix size of $256 \times 256$, one NEX, and the acquisition time of $40 \mathrm{~s}$ ) to measure trunk and glutei muscles. Transverse slices were acquired from the xyphoid process to the femoral heads ( $0.8 \mathrm{~cm}$ thick, $0.4 \mathrm{~cm}$ apart) $[7,11,18,20]$. A series of two stacks were acquired, using L4-L5 as a separating point. The umbilicus was located to identify the intervertebral space between L4 and L5 after acquisition of a localizer sequence. To reduce respiratory-motion artifact, participants were asked to inhale deeply and hold their breaths for 10-15 s. Two sets of 9-12 slices were captured, the first set extended superiorly from L4-L5 to the xyphoid process and the second set extended distally from L4 to L5 to the femoral heads $[7,11,18,20]$. 
Table 2 Mean \pm SD of individual muscles, IMF CSAs $\left(\mathrm{cm}^{2}\right)$, between-group differences and 95\% confidence interval (CI) at BL and PI for the TRT + RT and TRT groups

\begin{tabular}{|c|c|c|c|c|c|c|c|}
\hline \multirow[b]{2}{*}{ Regions } & \multirow[b]{2}{*}{ Variables } & \multicolumn{2}{|l|}{$\mathrm{TRT}+\mathrm{RT}$} & \multicolumn{4}{|l|}{ TRT } \\
\hline & & $\mathrm{BL} n=11$ & PI $n=10$ & $\begin{array}{l}\text { BL } n=10 \\
\text { (glutei } n=9 \text { ) }\end{array}$ & $\begin{array}{l}\text { PI } n=10 \\
(\text { legs } n=9 \text { ) }\end{array}$ & $\begin{array}{l}\text { Mean between-group } \\
\text { differences }\end{array}$ & $\begin{array}{l}\text { 95\% CI between-group } \\
\text { differences }\end{array}$ \\
\hline \multirow[t]{9}{*}{ Glutei } & Total GMX & $23.3 \pm 8.0$ & $26.6 \pm 9.4^{\mathrm{a}}$ & $27.2 \pm 6.0$ & $27.7 \pm 6.2$ & 2.59 & $(-0.04,5.24)$ \\
\hline & Absolute GMX & $21.8 \pm 7.9$ & $25.7 \pm 9.4^{\mathrm{a}}$ & $26.3 \pm 5.1$ & $26.9 \pm 6.4$ & $2.86^{\mathrm{b}}$ & $(0.30,5.45)$ \\
\hline & GMX IMF & $1.3 \pm 1.2$ & $0.8 \pm 0.7$ & $1.3 \pm 1.1$ & $0.8 \pm 0.6$ & 0.03 & $(-1.09,1.06)$ \\
\hline & Total GMD & $23.9 \pm 7.0$ & $26.8 \pm 8.0^{\mathrm{a}}$ & $26.3 \pm 4.2$ & $26.2 \pm 5.2$ & $1.89^{\mathrm{b}}$ & $(0.23,3.58)$ \\
\hline & Absolute GMD & $22.6 \pm 7.3$ & $25.8 \pm 7.9^{\mathrm{a}}$ & $24.8 \pm 4.5$ & $25.0 \pm 5.6$ & 1.54 & $(-0.45,3.57)$ \\
\hline & GMD IMF & $1.4 \pm 1.4$ & $1.0 \pm 0.9$ & $1.5 \pm 1.9$ & $1.2 \pm 1.1$ & 0.17 & $(-0.86,1.17)$ \\
\hline & GTOT & $67.0 \pm 17.5$ & $73.2 \pm 22.0^{\mathrm{a}}$ & $75.2 \pm 7.0$ & $74.2 \pm 10$ & $5.27^{\mathrm{b}}$ & $(0.90,9.69)$ \\
\hline & Absolute GTOT & $56.7 \pm 15.6$ & $64.3 \pm 18.7^{\mathrm{a}}$ & $65.1 \pm 7.5$ & $66.1 \pm 11.2$ & 4.56 & $(-0.23,9.42)$ \\
\hline & GTOT IMF & $10.2 \pm 5.9$ & $8.8 \pm 5.5$ & $10.1 \pm 3.7$ & $8.0 \pm 4.6$ & 0.9 & $(-1.97,3.71)$ \\
\hline \multirow[t]{11}{*}{ Legs } & Prox SOL & $14.3 \pm 5.0$ & $15.1 \pm 5.0$ & $14.4 \pm 3.1$ & $15.1 \pm 3.4$ & -0.31 & $(-1.93,1.33)$ \\
\hline & Mid SOL & $15.0 \pm 4.3$ & $15.2 \pm 3.9$ & $15.3 \pm 5.1$ & $15.9 \pm 5.0$ & -0.87 & $(-2.51,0.79)$ \\
\hline & Dist SOL & $7.8 \pm 2.2$ & $7.5 \pm 2.1$ & $10.1 \pm 4.3$ & $9.6 \pm 5.0$ & -0.02 & $(-1.08,1.03)$ \\
\hline & Prox GASTROC & $14.1 \pm 3.8$ & $13.7 \pm 3.7$ & $14.1 \pm 5.1$ & $13.7 \pm 4.9$ & -0.46 & $(-1.90,0.99)$ \\
\hline & Dist GASTROC & $9.2 \pm 2.8$ & $9.5 \pm 2.8$ & $9.3 \pm 4.9$ & $9.5 \pm 4.3$ & -0.10 & $(-1.07,0.87)$ \\
\hline & Prox TA & $4.7 \pm 1.1$ & $5.1 \pm 1.2$ & $4.6 \pm 1.0$ & $4.8 \pm 1.0$ & 0.40 & $(-0.18,0.97)$ \\
\hline & Mid TA & $5.9 \pm 1.6$ & $5.9 \pm 1.5$ & $5.7 \pm 1.6$ & $5.8 \pm 1.7$ & -0.05 & $(-0.37,0.26)$ \\
\hline & Dist TA & $3.0 \pm 1.0$ & $3.1 \pm 0.9$ & $3.4 \pm 1.4$ & $3.2 \pm 1.1$ & 0.28 & $(-0.22,0.78)$ \\
\hline & Prox TLM & $52.5 \pm 12.7$ & $52.6 \pm 11.8$ & $51.5 \pm 9.4$ & $51.7 \pm 10.2$ & -1.52 & $(-4.37,1.36)$ \\
\hline & Mid TLM & $45.4 \pm 11$ & $45.0 \pm 9.7$ & $47.1 \pm 15.2$ & $44.9 \pm 14.5$ & 0.56 & $(-2.95,-2.55)$ \\
\hline & Dist TLM & $29.6 \pm 6.8$ & $29.6 \pm 7.1$ & $31.3 \pm 11.6$ & $30.5 \pm 11$ & 0.19 & $(-2.55,2.95)$ \\
\hline \multirow[t]{6}{*}{ Trunk } & ES & $13.7 \pm 4.1$ & $14.2 \pm 4.0$ & $14.1 \pm 3.3$ & $14.4 \pm 2.5$ & 0.20 & $(-1.29,1.68)$ \\
\hline & $\mathrm{MF}$ & $5.5 \pm 1.6$ & $5.8 \pm 2.1$ & $6.0 \pm 1.4$ & $6.0 \pm 2.4$ & 0.18 & $(-0.75,1.11)$ \\
\hline & QL & $4.7 \pm 1.7$ & $5.2 \pm 2.1$ & $5.2 \pm 2.5$ & $4.8 \pm 2.1$ & 0.76 & $(-0.03,1.56)$ \\
\hline & PS & $8.1 \pm 3.1$ & $8.7 \pm 2.1$ & $7.7 \pm 2.8$ & $8.1 \pm 3.1$ & -0.21 & $(-1.05,0.63)$ \\
\hline & EIO & $20.1 \pm 5.5$ & $20.6 \pm 5.7$ & $19.1 \pm 7.2$ & $18.2 \pm 6.5$ & 1.41 & $(-0.14,2.96)$ \\
\hline & RA & $6.7 \pm 1.5$ & $7.2 \pm 2$ & $5.3 \pm 2.8$ & $5.5 \pm 2.5$ & 0.37 & $(-0.54,1.27)$ \\
\hline
\end{tabular}

$B L$ baseline, $P I$ post intervention 1, $R T$ resistance training, $T R T$ testosterone replacement therapy, $G M X$ gluteus maximus, $G M D$ gluteus medius, GTOT total gluts, IMF inter-muscular fat, SOL soleus, GASTROC gastrocnemius, TA tibialis anterior, TLM total leg muscles, ES erector spinae, $M F$ multifidus, $Q L$ quadratus lumborum, $P S$ iliopsoas, EIO external and internal obliques, $R A$ rectus abdominis, Prox proximal, Mid middle, Dist distal

${ }^{\mathrm{a}}$ Within group differences in the TRT + RT group; $P<0.05$

${ }^{\mathrm{b}}$ Mean between-group differences, $P<0.05$

To capture bilateral lower-leg muscles, transaxial images ( $8 \mathrm{~mm}$ thick, $15 \mathrm{~mm}$ apart) were captured from the knee to the ankle joints using a localized GE body array flex coil to ensure an adequate signal-to-noise ratio and higher image resolution (repetition time, $850-1000 \mathrm{~ms}$; echo time, $6.7 \mathrm{~ms}$; field of view, $20 \mathrm{~cm}$; matrix, $256 \times 256$ ) [15]. The coil was placed at the proximal border of the patella and extended to cover the entire leg [15].

Images were analyzed in a blinded fashion to the group assignment using a specialized imaging software (Win Vessel 2, Ronald Meyer, MSU, MI, USA). Using Image Jsoftware, images were matched between BL and PI based on bony landmarks. Images were automatically segmented into fat, skeletal muscle, and background/bone (high, mid and low intensity, respectively). To correct for intensity variations caused by radio frequency heterogeneity, a first pass segmentation was used. The corrected image was then re-segmented to the three intensity components using a fuzzy c-mean clustering algorithm $[3,11]$. For each selected image, the anatomical region of interest was manually traced pixel-by-pixel to quantify CSA $\left(\mathrm{cm}^{2}\right)$. Absolute IMF CSA was then quantified using a bimodal histogram at the midpoint between the muscle and fat peaks $[3,11,15]$. Each muscle group was presented as total CSA (muscle CSA without subtracting IMF) and absolute muscle CSA (muscle CSA after subtracting IMF). 
Fig. 1 Representative MRI images of the analyzed muscle groups which include: gluteus maximus (1a), gluteus minimus (2a), total glutei (b), erector spinae (1c), multifidus (2c), quadratus lumborum (3c), iliopsoas (4c), rectus abdominis (5c), external and internal obliques $(6 \mathbf{c})$, tibialis anterior (1d), soleus (2d), gastrocnemius (3d) and total leg muscles (d)
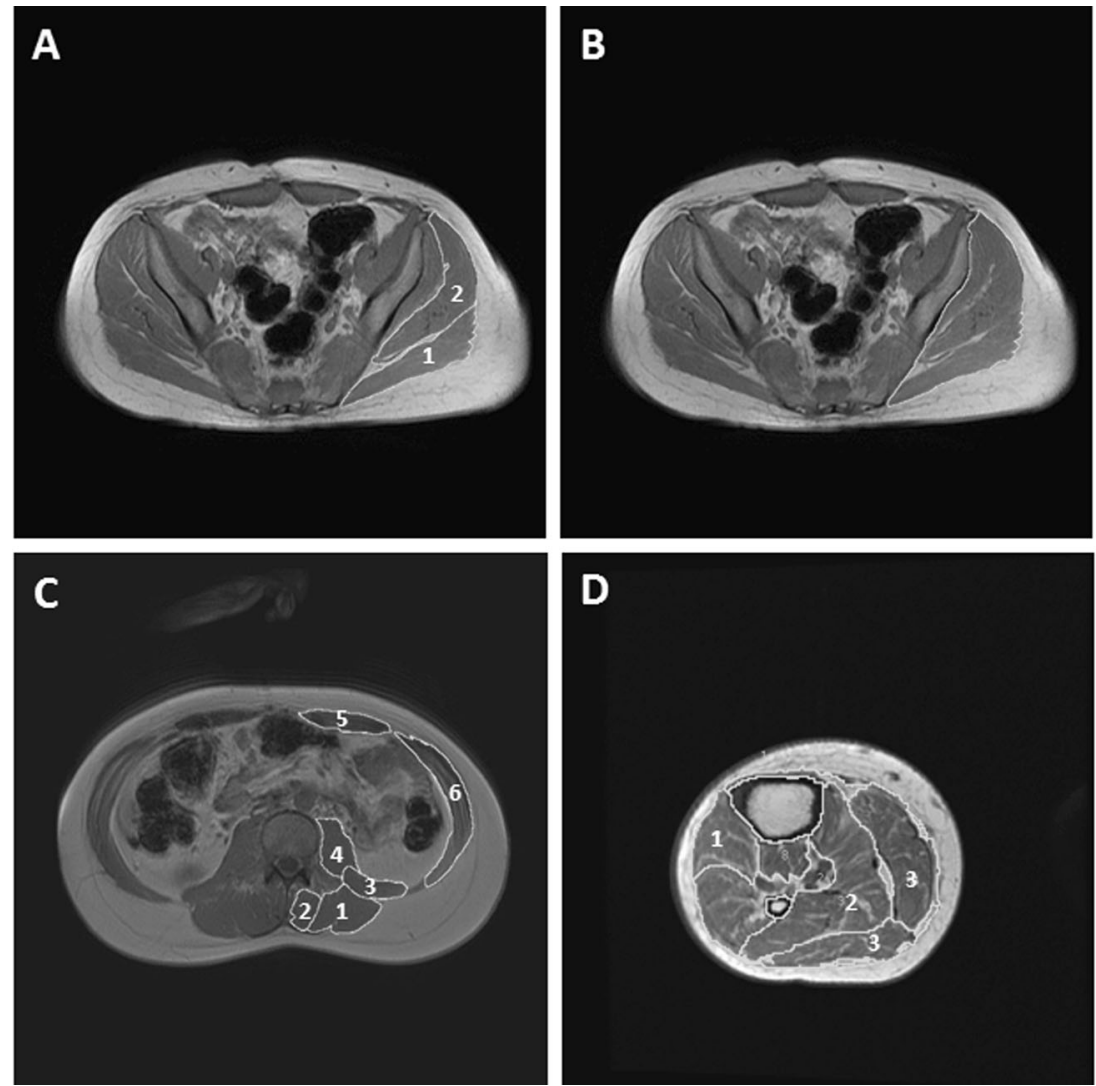

\section{Trunk muscles}

Six to seven images were selected between the upper lobes of the kidneys and the top of the iliac crest $[12,18]$. The images were selected to cover the primary trunk muscles as has been previously indicated $[12,18]$. The muscles that were manually traced are presented in Fig. 1c, and include the erector spinae (ES; 1), multifidus (MF; 2), quadratus lumborum (QL; 3), iliopsoas (PS; 4), rectus abdominis (RA; 5), and the external and internal obliques (EIO; 6) [18].

\section{Glutei muscles}

Four images were selected between the iliac crest and the top of the femur. The images were selected based on anatomical distinction of the boundaries of the glutei muscles and overall image quality. The left or the right glutei were analyzed based on the optimal quality of the signal intensity. The muscles that were manually traced and analyzed are presented in Fig. 1a, b. These include the gluteus maximus (GMX; A1), gluteus medius (GMD; A2) and total glutei $(\mathrm{GTOT}=\mathrm{GMX}+\mathrm{GMD}+$ gluteus minimus; $\mathrm{B})$. Muscle CSA and IMF CSA values were averaged across all four images.

\section{Lower leg muscles}

Twelve to fifteen images were selected between the knee and the ankle joints based on image quality. The regions that were manually traced are shown in Fig. 1d and include the TA; 1, SOL; 2, GAS; 3, and total leg muscles (TLM; entire traced region excluding bone). The leg selected for the analysis was determined based on the side chosen for glutei analysis. The analyzed images of the leg muscles were divided equally into three groups (proximal, middle, and distal) CSAs and the slices within each group were then averaged.

\section{Data analysis}

Participant characteristics were summarized using means and standard deviations (SD) or frequencies or percentages. All data are presented as mean \pm SD. Separate means and SD of each of the cardiometabolic risk factors were summarized by treatment group and time. To determine within time (BL vs. $\mathrm{PI}$ ) and between-group (TRT + RT vs. TRT only) differences, a $2 \times 2$ repeated measures ANOVA tests were performed. This model included fixed effects for both factors, as well as the interaction, and a random effect to account for dependence between the measures for a single individual. Statistical significance was set at $\alpha<0.05$ and due to the secondary focus of 
these outcomes in the clinical trial, no multiple adjustment correction was made [23]. The statistical software package $\mathrm{R}$ (v3.4.1) was used to perform all statistical analysis.

\section{Results}

\section{Participant characteristics}

The results presented are a follow-up analysis to our recently published work examining the effects of low-dose TRT + RT versus low-dose TRT only on cardiometabolic risk factors in the persons with SCI [11]. All participants received low-dose TRT from 2 to $6 \mathrm{mg}$ per day for 16 weeks and serum testosterone levels increased from 413.5 to $525 \mathrm{ng} / \mathrm{dl}$ in both groups [11]. There were no significant differences in the physical characteristics between the TRT + RT and TRT groups $(P>0.1$; Table 1$)$. One participant in the TRT + RT group withdrew in week 8 due to the recurring episodes of syncope, therefore PI MRI images were not captured. In the TRT only group, MRI was not captured in one participant due to the existence of remaining bullet fragments in his spine. Another two participants in the TRT group lacked optimal quality images of the glutei and lower leg muscles, therefore analysis was not performed. Glutei IMF data for two participants, one in the $\mathrm{TRT}+\mathrm{RT}$ and one in the TRT group, were not measured due to poor signal intensity quality, which resulted in difficulty separating pixels of the muscles from those of IMF.

\section{Trunk muscles}

Table 2 summarizes the CSAs of the trunk muscles in the $\mathrm{TRT}+\mathrm{RT}$ and TRT only groups at BL and PI with $95 \% \mathrm{CI}$. TRT + RT and TRT only did not induce changes $(P>0.05)$ in muscle CSAs of any of the trunk muscles [ES, MF, QL, IP, RA, and EIO]. Furthermore, there was no difference between groups. However, notable differences in the change scores were observed between TRT + RT and TRT for QL $(P=0.078)$ and EIO $(P=0.093)$.

\section{Glutei muscles}

Table 2 displays the CSAs of total glutei, absolute glutei (i.e., after subtracting IMF) and IMF CSAs at different time points (BL and PI) following both interventions (TRT $+\mathrm{RT}$ vs. TRT only), with the mean between-group differences and $95 \% \mathrm{CI}$ for each individual muscle.

\section{Total and absolute gluteus maximus muscle}

Total gluteus maximus CSA increased by $14 \%(P=0.003)$ in the TRT + RT group; however, there was no changes in the TRT group $(P=0.555 ;$ Fig. $2 \mathrm{a})$. TRT + RT resulted in a nominally greater increases in the gluteus maximus CSA with a mean difference of $2.59 \mathrm{~cm}^{2}$ compared with TRT only [95\% CI: $\left.-0.04,5.24 \mathrm{~cm}^{2} ; P=0.072\right]$. There was a $16 \%$ increase in absolute gluteus maximus for the TRT + RT group $(P=0.001)$ without changes in the TRT group $(P=0.496$; Fig. $2 \mathrm{~b}) . \mathrm{TRT}+\mathrm{RT}$ produced a greater increase $(P=0.044)$ in absolute gluteus maximus $m$. compared with TRT only group with a mean between-group differences of $2.86 \mathrm{~cm}^{2}$ and $95 \% \mathrm{CI}: 0.30,5.45 \mathrm{~cm}^{2}$. However, gluteus maximus IMF did not change in either intervention.

\section{Total and absolute gluteus medius muscle}

A $10 \%$ increase in the total gluteus medius CSA was observed for the TRT + RT group $(P=0.001)$ but not for the TRT group $(P=0.372)$. TRT + RT produced a greater increase $(P=0.042)$ in total gluteus medius $\mathrm{m}$. compared with TRT only group with a mean between-group differences of $1.89 \mathrm{~cm}^{2}$ and $95 \%$ CI: $0.23,3.58 \mathrm{~cm}^{2}$.

The absolute gluteus medius CSA increased by $14 \%$ in the TRT + RT group $(P=0.006)$, but not for the TRT group $(P=0.173)$. No differences in absolute glutes medius CSA were observed between both groups $(P=0.153)$ (Fig. 2d).

\section{Total and absolute whole glutei muscle}

Total glutei $\mathrm{m}$. increased $(P=0.02)$ for both groups from BL to PI with an interaction between the TRT $+\mathrm{RT}$ and the TRT groups $(P=0.04)$. The TRT $+\mathrm{RT}$ group showed an $8 \%$ increase $(P=0.01)$ in the total glutei CSA (Fig. 2e). TRT + RT produced a greater increase $(P=0.035)$ in total glutei $\mathrm{m}$. compared with TRT only group with a mean between-group differences of $5.27 \mathrm{~cm}^{2}$ and $95 \%$ CI: $0.90,9.69 \mathrm{~cm}^{2}$.

Absolute total glutei CSA increased $(P=0.004)$ for both groups from BL to PI. Absolute total glutei CSA increased by $11 \%(P=0.005)$ for the TRT + RT group without changes in the TRT group $(P=0.3$; Fig. $2 \mathrm{f})$. There was a trend towards an interaction between the two groups $(P=$ 0.09). TRT + RT produced a nominal increase $(P=0.09)$ in absolute glutei $\mathrm{m}$. compared with TRT only group with a mean between-group differences of $4.56 \mathrm{~cm}^{2}$ and $95 \%$ CI: $-0.23,9.42 \mathrm{~cm}^{2}$.

\section{Lower-leg muscles}

The CSA values for the leg muscles are summarized in Table 2 for the TRT + RT and TRT groups at BL and PI. There were no observed differences between the two groups and changes from BL to PI following either TRT + RT or TRT for the proximal, middle and distal TA m., SOL m., GASs m., and TLM. 


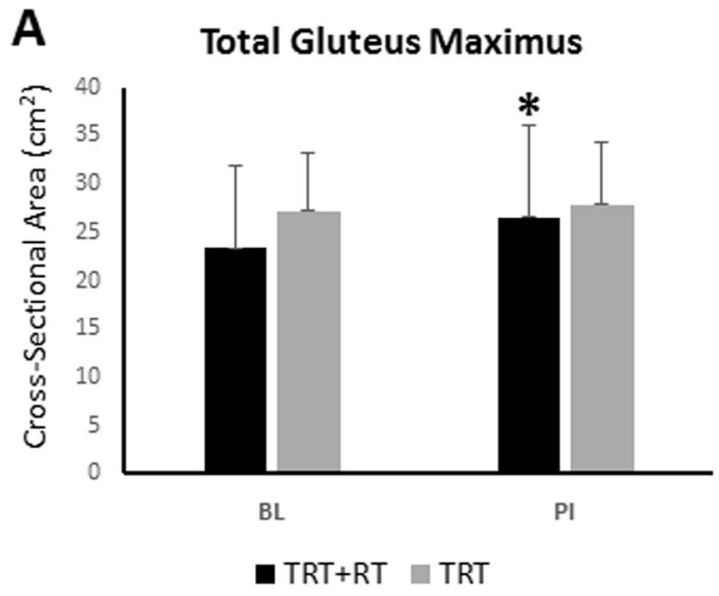

C

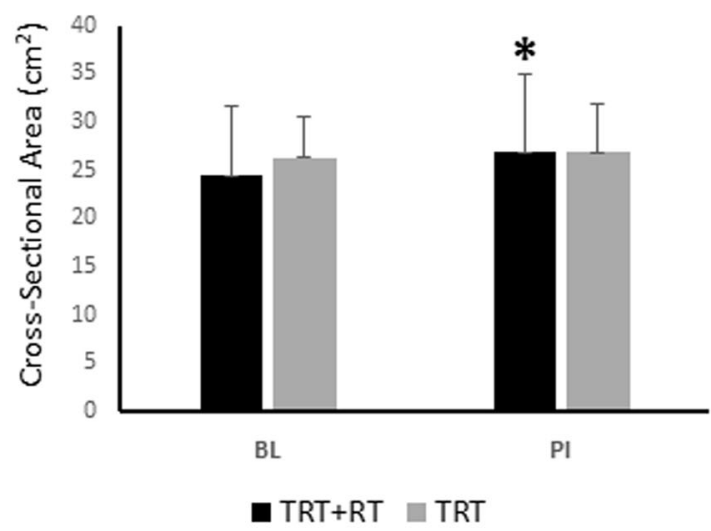

E

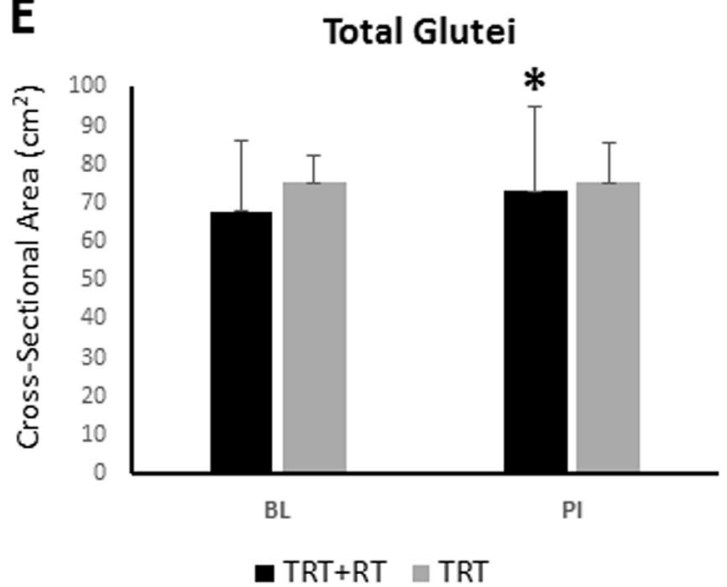

B

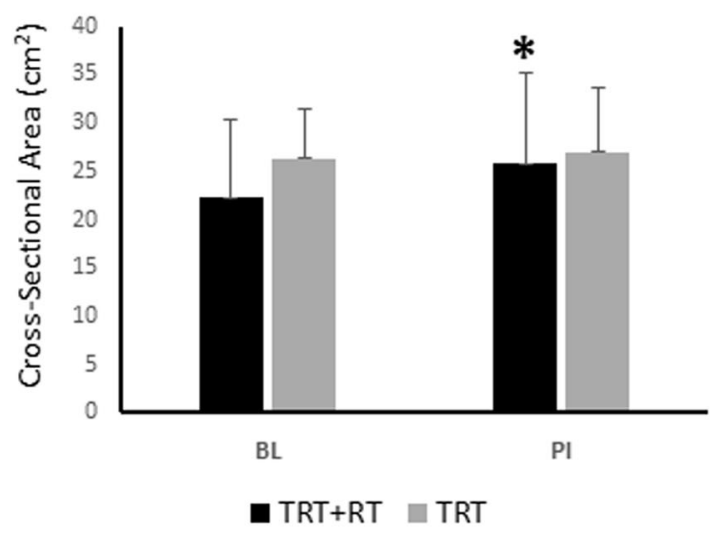

D

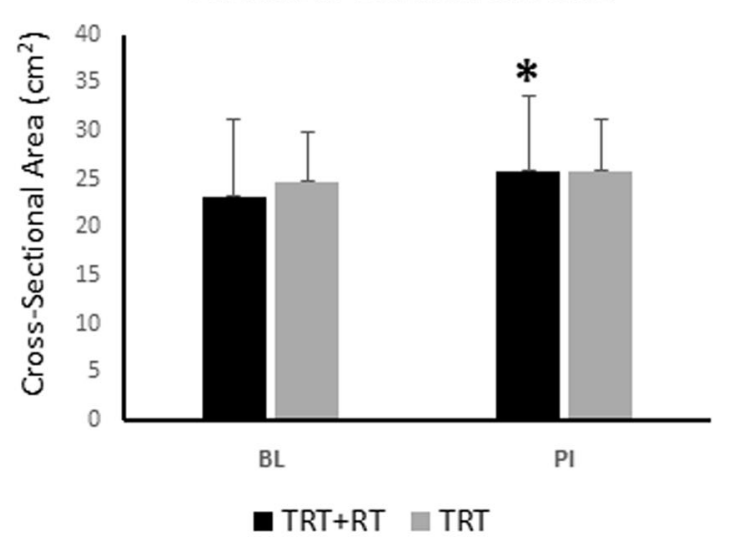

$\mathbf{F}$

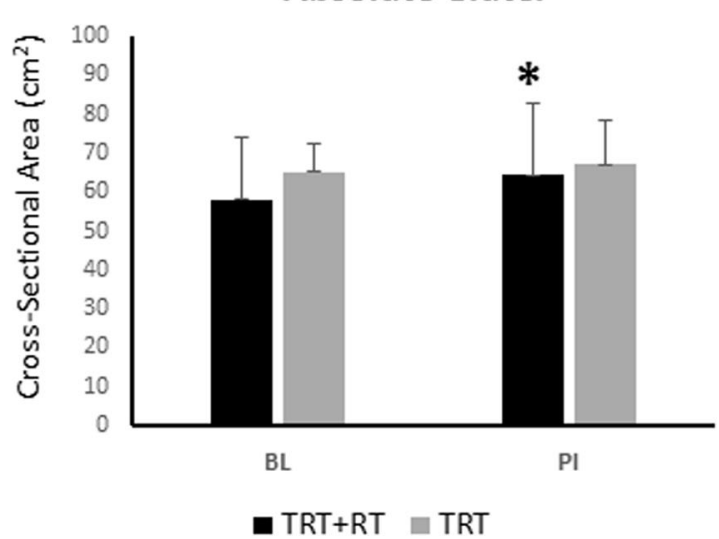

Fig. 2 The mean \pm SD of total and absolute gluteus maxiumus $(\mathbf{a}, \mathbf{b})$, minimus $(\mathbf{c}, \mathbf{d})$, and total muscle $(\mathbf{e}, \mathbf{f}) \mathrm{CSA}$ for the TRT $+\mathrm{RT}$ and the TRT only group during BL and PI. Asterisk significant change in muscle CSA at $P<0.05$

\section{Discussion}

The major findings of this secondary pilot study indicated that the addition of low-dose TRT to RT induced the hypertrophy of nontargeted untrained glutei muscles. Mean between-group differences suggested that TRT + RT resulted in greater increases in glutei m. CSA compared with the TRT only. Although trunk muscles were not significantly affected, a trend towards an interaction between the two groups for the QL and EIO may indicate that these muscles are positively affected in the TRT $+\mathrm{RT}$ group compared with the TRT only group. Neither TRT + RT nor 
TRT alone increased CSA of the untrained lower-leg muscles. Furthermore, the combined application of RT and low-dose TRT may be recommended as a future prophylactic intervention for the prevention of pressure injuries and improvement of body composition because of its positive effects on the trained as well as untrained muscle groups.

\section{Rationale of studying untrained muscles}

The rationale of studying the untrained muscles was based on previous work that demonstrated the effects of unilateral RT on contralateral untrained muscles [24]. We have previously shown that 8 weeks of unilateral NMES-RT did not result in contralateral muscle hypertrophy of the untrained knee extensor muscle group [13]. The concept of evoking muscle hypertrophy in untrained muscles may be beneficial to those with sacral ulcers greater than grade II, in which sitting may induce shear stress or direct applications of electrical stimulation on the exposed ulcer may not be feasible. Biomechanically, it is also difficult to load the hip extensors to evoke hypertrophy because of the long lever arm of the lower extremity and difficulty of attaining prone position for extended period after SCI.

\section{Potential mechanisms of evoking hypertrophy in the untrained muscles}

The mechanism behind the hypertrophy of untrained muscles still remains unclear; however, there are several hypotheses that may explain these findings. During training of the knee extensors, possible bleeding of the electrical current to the glutei muscles may have occurred due to the smaller muscle size after SCI. In addition, there may be occurrence of a volley phenomenon after using a long pulse duration of $1 \mathrm{~ms}$ and lead to anti-dromic afferent stimulation of knee extensors [25]. This afferent stimulation may activate the alpha motor neuron pools and lead to indirect stimulation of the adjacent untrained muscle groups. Despite the fact that we used a pulse duration of $450 \mu \mathrm{s}$, increasing the current amplitude to stimulate the knee extensors reflexively resulted in noticeable hip flexion and ankle dorsiflexion in several of our participants; suggesting the possibility of anti-dromic volley stimulation of the untrained muscles. Moreover, isometric torque data at $100 \mathrm{~mA}$ and $30 \mathrm{~Hz}$ suggested the occurrence of the volley phenomenon during examining knee extensor peak torque in response to either intervention (data not shown). Gorgey et al. also noted a significant increase in IGF-1 following 12 weeks of NMES-RT in men with SCI. This increase was also associated with hypertrophy of the knee extensors [7]. However, in the current trial, we noticed a decrease in IGF1 and increase in insulin growth factors binding protein-3 following 16 weeks of training [11]. The discrepancy between both studies may either attribute to the influence of TRT on insulin growth factors or due to the duration of both studies (12 vs. 16 weeks) [11].

\section{Clinical relevance of hypertrophy of the glutei muscles}

Our BL measurements of gluteus maximums m. agreed with a previous report using CT scans in persons with chronic $\mathrm{SCI}$ [17]. Wu et al. showed that gluteus maximus $\mathrm{m}$. in persons with SCI was 55\% of that of able-bodied controls [17]. Furthermore, the authors highlighted that muscle quality has dramatically changed by increasing infiltration of IMF (SCI: 29 vs. AB: 8\%) [17]. Therefore, TRT + RT is likely to reciprocate this profile by increasing glutei muscle size, improving intrinsic tissue health, enhancing regional blood flow and preventing the frequent occurrence of pressure injuries in this population. Dolbow et al. studied seat pressure changes in men with SCI after 8 weeks of functional electrical stimulation cycling [26]. The study showed a positive trend towards a reduction in ischial seat pressure [26]. Our findings indicate that this trend may result from increasing glutei CSA noted in the current work. Eight weeks of NMES helped to improve tissue oxygenation levels and seating pressure distribution in individuals with SCI [16]. Increases in muscle size may have been the underlying mechanism for previous findings [16, 26], as muscle helps to improve surrounding tissue health through increased vascularization and mitochondrial oxygen utilization. These are all critical factors in preventing costly and potentially life-threatening pressure injuries after SCI.

In elderly population, an increase in knee extensor CSA by $5-15 \%$ following RT for $6-30$ weeks is considered clinically relevant [27]. Wu et al. demonstrated small $5-11 \%$ increases in sacral interphase pressures following applications of implanted electrical stimulation system for 5 min [28]. Therefore, the increase in glutei muscle CSA $(8-16 \%)$ is a noteworthy finding, and may lead to a number of important health benefits including increasing tissue interphase pressures and tissue oxygenation in individuals with SCI. We recently showed that 16 weeks of low-dose TRT + RT led to an increase in total and absolute thigh muscle CSA by roughly $20 \mathrm{~cm}^{2}$, while no changes in muscle size were noted following low-dose TRT only [11]. Our findings suggest that the addition of RT to low-dose TRT may be beneficial in inducing muscle hypertrophy of the trained thigh muscles and adjacent glutei muscles. Higher serum testosterone in men with SCI is linked to positive body composition [18] and cardiometabolic outcomes [29]. However, low-dose TRT administered in the current study was not enough to induce trunk, glutei or lower leg muscle hypertrophy. 


\section{Why study trunk muscles?}

Exercise involving NMES and functional electrical stimulation has primarily focused on the lower and upper extremity muscle groups. Trunk fat-free mass represents $49-51 \%$ of total body fat-free mass [30] and is essential for posture, transfers, coughing, and breathing [31]. In addition, trunk muscles have been associated with a positive metabolic profile and negatively related to central adiposity [18]. Gorgey et al. investigated the effects of 12 weeks of NMES on the CSA of adjacent trunk muscles and concluded that trunk muscles were not affected by NMES-RT of the thigh muscles [12]. Our results showed a trend towards an interaction between the low-dose TRT + RT and TRT group, which may indicate that the addition of TRT to RT helps to amplify the effects of RT. Bauman et al. showed increase in trunk lean mass following 12 months of administering TRT in hypogonadal men with SCI [22]. The lack of robust changes in the size of trunk muscles may be explained by the fact the trunk muscle mass collectively is only $1.2 \mathrm{~kg}$ [30] and the large mass of the surrounding visceral and subcutaneous adipose tissue may impede the progression of the current to directly activate them. The current findings may also suggest that direct stimulation of the trunk muscles is necessary to yield muscle hypertrophy in persons with SCI. A future clinical larger scale trial may reveal such interaction noted with the trunk muscles.

\section{MRI as an imaging technique}

MRI captures multi-axial slices across the entire length of the muscle [15]. This provides the robustness of comparing muscle CSA at the same location between BL and PI as well as accurate quantification of absolute muscle CSA after separation of IMF [3, 7-11]. Furthermore, the use of a localized coil improved signal-to-noise ratio and greatly enhance image quality; which further helps in easily matching images based on bony landmarks between BL and PI. The improvement in mage quality, accurate matching of images at different time points as well as capturing and analyzing images in a blinded fashion reduce the likelihood of introducing error from using different location or orientation.

\section{Limitations}

The age range was only limited to $18-50$ years to ensure safety and avoid potential cardiovascular risk consequences that may result from using TRT. Only men were recruited with motor complete SCI (AIS A or B) and women were not enrolled for ethical consideration regarding using TRT.

Because of budgetary constraints, the design of the study did not include NMES-RT only or TRT placebo-controlled groups. The NMES-RT effects on muscle size have been widely studied on the trained knee extensor and untrained trunk muscle groups in persons with motor complete SCI [7, 11-14]. Five different studies have clearly demonstrated $35-40 \%$ hypertrophy of the knee extensor muscle group following NMES-RT in persons with motor complete SCI [7, 8, 10,11, 13]. However, NMES-RT was ineffective in evoking changes in the untrained trunk muscles. The rationale behind the current design (TRT $+\mathrm{RT}$ vs. TRT only) was to introduce an alternative rehabilitation approach that can promote muscle hypertrophy or increase lean mass in pre-existing medical conditions that may be inadequate to participate in NMES-RT program; because of low tolerance to electrical stimulation with intact sensation below the level of injury, autonomic dysreflexia, pressure ulcers greater than grade II, and peripheral lower motor neuron injury.

In addition, few participants were not included in the final analysis because of specific precautions regarding using MRI or because of low-image quality. The overall final sample size may have impacted the findings of the current study. A larger sample would be ideal to account for the attrition rate or failure to capture MRI in persons with SCI. It is likely that the trunk muscles were impacted due to the trend towards an interaction between the two study groups, however these results may have been masked by the heterogeneity of our study sample. According to Rankin et al., individuals with tetraplegia have $13 \%$ smaller trunk muscle CSA than individuals with paraplegia [30]. Our sample included persons with tetraplegia and paraplegia; suggesting that the effects of training may have been masked.

The use of low-dose TRT may not suffice to evoke necessary changes in muscle size. Our participants received 2-6 $\mathrm{mg}$ of testosterone (average of $4 \mathrm{mg}$ per day) over a 4-month period [11]. Bauman et al. used a dose of 5-10 mg per day in hypogonadal men SCI and showed increase in both lean mass and basal metabolic rate following 12 months [22]. Nightingale et al. recommended an optimum dose of 6-8 mg per day to increase lean mass in men with SCI [21].

Considering the cost and time of conducting randomized clinical trials, additional data on untrained muscle groups, spasticity, bone architecture, and FES-cycling peak $\mathrm{VO}_{2}$ were intentionally collected. The original study was not powered based on these outcome variables and possibly type II error (1- $\beta$ ) was committed. The current study may have lacked the necessary power to demonstrate statistical changes in both the trunk and leg muscles. Our main clinical trial was primarily directed towards studying the effects of low-dose TRT + RT versus TRT only on muscle size, body composition and visceral adiposity (primary outcome variables) and metabolic profile as determined by the 
changes in basal metabolic rate, carbohydrate, lipid, and inflammatory biomarkers (secondary outcome variables) [11]. Therefore, the findings of the current trial should be treated with caution; because the work is considered a secondary analysis of the untrained muscles; which was not the primary focus of our concluded clinal trial [11]. The findings may be considered as exploratory pilot work that can be effectively used to calculate effect sizes for untrained muscles to adequately power future clinical trials. Pilot studies are of substantial value to gain feasibility and a preliminary indicator of adverse events [32]. The two groups were matched at $\mathrm{BL}$ and adherence to therapies exceeded $95 \%$ with very minimal side effects. The preliminary results are promising and a larger powered randomized placebo-controlled trial may be warranted to test the hypothesis and provide firm scientific conclusions.

\section{Conclusion}

The pilot findings support that 16 weeks of low-dose TRT + RT leads to hypertrophy of untrained glutei muscles, which may be of health-related significance considering the prevalence of pressure injuries in this population. The findings support between-group differences that favor the effects of TRT + RT compared with the TRT only on the glutei muscles. In addition, there was a trend towards an interaction between the two groups in the QL and EIO trunk muscles, and no effect was seen on the lower-leg muscles. The findings may suggest the need to directly stimulate the trunk muscles or administering a higher dose of TRT to achieve reasonable changes in the size of the trunk muscles. Furthermore, applying and evaluating novel combinatory therapies is warranted given the heterogenous SCI population and the limited effectiveness of single therapies. Overall, these findings may help developing future exercise protocols that can be used to prevent pressure injuries and increase muscle size, thereby improving muscle quality and overall cardiometabolic health in persons with SCI.

\section{Data archiving}

The datasets generated during and/or analyzed during the current study are not publicly available due to data restriction policy by the Department of Veteran Affairs but are available from the corresponding author on reasonable request.

Acknowledgements We would like to thank R. Chase Wade and Ryan M. Sumrell for helping with capturing and analysis of data. We want to thank all of the participants in our study for their time and effort. We would also like to thank Hunter Holmes McGuire Research Institute and Spinal Cord Injury Services and Disorders for providing a location for us to conduct our clinical human research trials. Lastly, we would like to thank the VAMC Radiology Service for using their MRI for research purposes.

Funding The study is supported by the Department of Veteran Affairs, Veteran Health Administration, Rehabilitation Research and Development Service (B7867-W (ASG), B9212-C and B2020-C); clinical trial registration number NCT01652040.

Author contributions ASG is responsible for designing the study, writing the protocol and report, conducting the search, extracting and analyzing data, interpreting results, assisted with writing, read, edit and approved the final version, and providing funds for the entire study. SMA is responsible for analyzing MRI images, data analysis and data writing, updating reference lists, and creating Summary of findings' tables; read and approved the final version. AS is a biostatistician and helped analyzing all the study outcomes. REK is responsible for recruitment and screening potentially eligible studies, trained patients, archive, and organize data. RK provided guidance on MRI analysis and MRI parameters; read and approved the final version. RAA provided medical monitoring to the SCI participants, examined serum testosterone, prescribe testosterone replacement therapy, assisted with writing, reading, editing and approving the final version.

\section{Compliance with ethical standards}

Statement of ethics We certify that all applicable institutional and governmental regulations concerning the ethical use of human volunteers were followed during the course of this research

Conflict of interest The authors declare that they have no conflict of interest.

Publisher's note Springer Nature remains neutral with regard to jurisdictional claims in published maps and institutional affiliations.

\section{References}

1. Martin Ginis KA, van der Scheer JW, Latimer-Cheung AE, et al. Evidence-based scientific exercise guidelines for adults with spinal cord injury: an update and a new guideline. Spinal Cord. 2018;56:308-21.

2. Castro MJ, Apple DF, Hillegass EA, Dudley GA. Influence of complete spinal cord injury on skeletal muscle cross-sectional area within the first 6 months of injury. Eur J Appl Physiol Occup Physiol. 1999;80:373-8.

3. Gorgey AS, Dudley GA. Skeletal muscle atrophy and increased intramuscular fat after incomplete spinal cord injury. Spinal Cord. 2007;45:304-9.

4. Spungen AM, Wang J, Pierson RN, Bauman WA. Soft tissue body composition differences in monozygotic twins discordant for spinal cord injury. J Appl Physiol. 2000;88:1310-5.

5. Bauman WA, Spungen AM. Coronary heart disease in individuals with spinal cord injury: assessment of risk factors. Spinal Cord. 2008;46:466-76.

6. Chromiak JA, Smedley B, Carpenter W, Brown R, Koh YS, Lamberth JG, et al. Effect of a 10-week strength training program and recovery drink on body composition, muscular strength and endurance, and anaerobic power and capacity. Nutrition. 2004;20: $420-7$.

7. Gorgey AS, Mather KJ, Cupp HR, Gater DR. Effects of resistance training on adiposity and metabolism after spinal cord injury. Med Sci Sports Exerc. 2012;44:165-74. 
8. Mahoney ET, Bickel CS, Elder C, Black C, Slade JM, Apple D Jr, et al. Changes in skeletal muscle size and glucose tolerance with electrically stimulated resistance training in subjects with chronic spinal cord injury. Arch Phys Med Rehabil. 2005;86:1502-4.

9. Ryan AS, Ivey FM, Prior S, Li G, Hafer-Macko C. Skeletal muscle hypertrophy and muscle myostatin reduction after resistive training in stroke survivors. Stroke. 2011;42:416-20.

10. Dudley GA, Castro MJ, Rogers S, Apple DF Jr. A simple means of increasing muscle size after spinal cord injury: a pilot study. Eur J Appl Physiol Occup Physiol. 1999;80:394-6.

11. Gorgey AS, Khalil RE, Gill R, Gater DR, Lavis TD, Cardozo C, et al. Low-dose testosterone and evoked resistance exercise after spinal cord injury [TEREX-SCI] on cardio-metabolic risk factors: an open-label randomized clinical trial. J Neurotrauma. 2019;36: 2631-45.

12. Gorgey AS, Dolbow DR, Cifu DX, Gater DR. Neuromuscular electrical stimulation attenuates thigh skeletal muscles atrophy but not trunk muscles after spinal cord injury. J Electromyogr Kinesiol. 2013;23:977-84.

13. Gorgey AS, Lester RM, Wade RC, Khalil RE, Khan RK, Anderson ML, et al. A feasibility pilot using telehealth videoconference monitoring of home-based NMES resistance training in persons with spinal cord injury. Spinal Cord Ser Cases. 2017;3:17039.

14. Gorgey AS, Khalil RE, Gill R, O'Brien LC, Lavis T, Castillo T, et al. Effects of testosterone and evoked resistance exercise after spinal cord injury (TEREX-SCI): study protocol for a randomised controlled trial. BMJ Open. 2017;7:e014125. https://doi.org/10. 1136/bmjopen-2016-014125.

15. Lester RM, Johnson K, Khalil RE, Khan R, Gorgey AS. MRI analysis and clinical significance of lower extremity muscle cross-sectional area after spinal cord injury. Neural Regen Res. 2017;12:714-22.

16. Bogie KM, Triolo RJ. Effects of regular use of neuromuscular electrical stimulation on tissue health. J Rehab Res Dev. 2003; 40:469-76.

17. Wu GA, Bogie KM. Not just quantity: gluteus maximus muscle characteristics in able-bodied and SCI individuals-implications for tissue viability. J Tissue Viability. 2013;22:74-82.

18. Abilmona SM, Gorgey AS. Associations of the trunk skeletal musculature and dietary intake to biomarkers of cardiometabolic health after spinal cord injury. Clin Physiol Funct Imaging. 2018;38:949-58.

19. Bauman WA, La Fountaine MF, Spungen AM. Age-related prevalence of low testosterone in men with spinal cord injury. J Spinal Cord Med. 2014;37:32-39.
20. Abilmona SM, Sumrell RM, Gill RS, Adler RA, Gorgey AS. Serum testosterone levels may influence body composition and cardiometabolic health in men with spinal cord injury. Spinal Cord. 2019;57:229-39.

21. Nightingale TE, Moore P, Harman J, Khalil R, Gill RS, Castillo T, et al. Body composition changes with testosterone replacement therapy following spinal cord injury and aging. A mini review. J. Spinal Cord Med. 2018;41:624-36.

22. Bauman WA, Cirnigliaro CM, La Fountaine MF, Jensen AM, Wecht JM, Kirshblum SC, et al. A small-scale clinical trial to determine the safety and efficacy of testosterone replacement therapy in hypogonadal men with spinal cord injury. Horm Metab Res. 2011;43:574-9.

23. Bender R, Lange S. Adjusting for multiple testing-when and how? J Clin Epidemiol. 2001;54:343-9.

24. Lee M, Carroll TJ. Cross education: possible mechanisms for the contralateral effects of unilateral resistance training. Sports Med. 2007;37:1-14.

25. Collins DF, Burke D, Gandevia SC. Sustained contractions produced by plateau-like behavior in human motoneurones. J Physiol. 2002;538:289-301.

26. Dolbow DR, Gorgey AS, Dolbow JD, Gater DR. Seat pressure changes after eight weeks of functional electrical stimulation cycling: a pilot study. Top Spinal Cord Inj Rehabil. 2013;19: 222-8.

27. Cadore EL, Pinto RS, Bottaro M, Izquierdo M. Strength and endurance training prescription in healthy and frail elderly. Aging Dis. 2014;5:183-95. 1

28. Wu GA, Lombardo L, Triolo RJ, Bogie KM. The effects of combined trunk and gluteal neuromuscular electrical stimulation on posture and tissue health in spinal cord injury. PM R. 2013;5:688-96.

29. Sullivan SD, Nash MS, Tefara E, Tinsley E, Groah S. Relationship between gonadal function and cardiometabolic risk in young men with chronic spinal cord injury. PM R. 2017;10: 373-81.

30. Rankin KS, O'Brien LC, Gorgey AS. Quantification of trunk and android lean mass using dual energy x-ray absorptiometry compared to magnetic resonance imaging after spinal cord injury. $\mathrm{J}$ Spinal Cord Med. 2018;20:1-9.

31. Granata KP, Wilson SE. Trunk posture and spinal stability. Clin Biomech. 2001;16:650-9.

32. Leon AC, Davis LL, Kraemer HC. The role and interpretation of pilot studies in clinical research. J Psychiatr Res. 2011;45:626-9. 